\title{
Existence of Viscous Profiles for the Compressible Navier-Stokes Equations
}

\author{
E. LORIN* \\ Departement de Mathematiques, Universite d'Orsay, Bat 425, 91405 Orsay, France
}

(Received 5 May 2003)

\begin{abstract}
In this article we show the existence of some particular solutions of the compressible Navier-Stokes equations called viscous profiles. The existence of such solutions provides an entropy criterion. The crucial point in the demonstration is the use of the center manifold theorem, and the main difficulty comes from the non-invertibility of the viscosity matrix in the Navier-Stokes equations.
\end{abstract}

Keywords: Viscous profiles; Center manifold theorem; Entropy condition; Navier-Stokes equations

AMS Subject Classifications: 35L65 (35B35)

\section{INTRODUCTION}

The aim of this article is to prove the existence of certain particular orbits which derive from a dynamical system called viscous profiles [2] issued of the monodimensional compressible Navier-Stokes equations. The introduction of such solutions is related to the frame of the selection of physical weak solutions (entropy solutions). It is, indeed, well known that the Lax shock condition, the Oleinik's condition or Liu's criterion [2] are all criterions of selecting entropy solutions (any of these conditions are totally relevant for treating all the possible configurations). Following this, the existence of viscous profiles provides another criterion to select physical solutions from all the weak solutions. We can reasonably consider that the good notion is "around" all the previous ones. In fact, we can show the equivalence of these different criterions, for example when the characteristic field is genuinely nonlinear; but when the characteristic field is linearly degenerate the equivalence does not occur anymore [4]. In addition to this, the existence is not sufficient to consider viscous profiles as physical solutions. Surely enough, it has to come with the structural stability of these profiles. In this case we will only consider the existence and not the stability. Nevertheless some results of stability can be found, for example, in [5]. This work is a generalization (with a different method) of [7], where the case of stationary shock layers is treated.

\footnotetext{
*E-mail: elorin@cs.umn.edu
} 
The principle of the demonstration is the following. The first step consists of reverting the partial differential system to a dynamical system, which is called the profile equation (2). The existence of heteroclinic orbits in the profile equation is the result to prove. The main difficulty is that the viscosity matrix is not invertible. For a scalar matrix, results of existence have been proved as shown [4]. The extension to any invertible dissipative (for the entropy of the system) matrices is proved in this book too. In order to avoid this particular difficulty we will project the system on a manifold on which the "new" system will possess an invertible dissipative matrix. The main issue will be the application of the center manifold theorem [3]. The searched heteroclinic orbits do belong to this particular manifold tangent to the center subspace of the dynamical system. In conclusion we will study the direction of the motion along the orbits with increasing time.

Note that a recent advancing has been done concerning the study of noninvertible diffusion matrix [6].

\section{EXISTENCE OF VISCOUS PROFILES}

We consider the compressible Navier-Stokes equations in Lagrangian coordinates:

$$
\left\{\begin{array}{l}
\tau_{t}-z_{x}=0 \\
z_{t}+p_{x}=\varepsilon\left(b(\tau, e) z_{x}\right)_{x}, \\
\left(e+\frac{1}{2} z^{2}\right)_{t}+(p z)_{x}=\varepsilon\left(k(\tau, e) e_{x}\right)_{x}+\varepsilon\left(b(\tau, e) z z_{x}\right)_{x} .
\end{array}\right.
$$

In (1) $\tau$ represents the specific volume, $z$ the speed of the fluid and $e$ the internal energy. At the end, we note $E=e+z^{2} / 2$, the total energy. Let $u_{g}$ and $u_{d}$ be a nonstationary $k$-shock with speed $\sigma$, solution of the Euler equations. We want to prove the existence of a heteroclinic orbit solution of the profile equation which is related to (1). We also suppose that the viscosity and the thermal conductivity respectively denoted by $b(\tau, e)$ and $k(\tau, e)$ are the following positive constants:

$$
\left\{\begin{array}{l}
b(\tau, e)=\mu>0, \\
k(\tau, e)=\kappa>0,
\end{array}\right.
$$

with $(\tau, e) \in \tilde{\mathcal{U}}$ and open set of $\subset\left(\mathbb{R}_{+}^{*}\right)^{2}$. We set in the sequel $\operatorname{Pr}=\mu / \kappa$ the Prandtl number. Supposing also that the pressure checks the perfect gas law: $p(\tau, e)=$ $(\gamma-1) e / \tau$ where $\gamma>1$. Considering a shock $\left(u_{g}, u_{d} ; \sigma\right)$, we search for a viscous profile (with $\sigma \neq 0$ ) i.e. a particular solution of the following type: a family functions $\left(u^{\varepsilon}\right)_{\varepsilon>0} \in C^{1}(\Omega, \mathcal{U})$, where $\mathcal{U}$ is an open set of $\mathbb{R}_{+}^{*} \times \mathbb{R} \times \mathbb{R}_{+}^{*}$ and $\Omega$ is an open set of $\mathbb{R}^{2}$ :

$$
u^{\varepsilon}(t, x)=U\left(\frac{x-\sigma t}{\varepsilon}\right)
$$

such that:

$$
\left\{\begin{array}{l}
U(-\infty)=u_{g} \\
U(+\infty)=u_{d}
\end{array}\right.
$$


The physical flux writes:

$$
\begin{gathered}
\left(f_{1}, f_{2}, f_{3}\right)^{t}: \mathcal{U} \subset \mathbb{R}_{+}^{*} \times \mathbb{R} \times \mathbb{R}_{+}^{*} \rightarrow \mathbb{R}^{3}, \\
\left(\begin{array}{c}
\tau \\
z \\
E
\end{array}\right) \mapsto\left(\begin{array}{c}
-z \\
p \\
p z
\end{array}\right) .
\end{gathered}
$$

When $\varepsilon$ tends to $0, u^{\varepsilon}(t, x)$ is given by the following function:

$$
u(t, x)= \begin{cases}u_{g}, & x<\sigma t \\ u_{d}, & x>\sigma t\end{cases}
$$

such that the Rankine-Hugoniot relation is checked. This shock is then considered as an admissible shock. Injecting $u^{\varepsilon}$ in the initial system gives the following differential system:

$$
\left(B(U) U^{\prime}\right)^{\prime}=(f(U))^{\prime}-\sigma U^{\prime} .
$$

Moreover,

$$
\lim _{\xi \rightarrow-\infty} B(U(\xi)) U^{\prime}(\xi)=\lim _{\xi \rightarrow+\infty} B(U(\xi)) U^{\prime}(\xi)=0
$$

Then, integrating (2) between ] $-\infty, \xi]$ we obtain a dynamical system called the profile equation:

$$
B(U) U^{\prime}=f(U)-f\left(u_{g}\right)-\sigma\left(U-u_{g}\right),
$$

where

$$
B(U)=\left(\begin{array}{ccc}
0 & 0 & 0 \\
0 & \mu & 0 \\
0 & (\mu-\kappa) z & \kappa
\end{array}\right)
$$

Recall that [7] treats the case $\sigma=0$. Futhermore the Jacobian taken in $U$ is given by:

$$
d f(U)=\left(\begin{array}{ccc}
0 & -1 & 0 \\
\frac{(1-\gamma) e}{\tau^{2}} & \frac{(1-\gamma) z}{\tau} & \frac{(\gamma-1)}{\tau} \\
\frac{(1-\gamma) e z}{\tau^{2}} & \frac{(1-\gamma) z^{2}}{\tau}+\frac{e(\gamma-1)}{\tau} & \frac{(\gamma-1) z}{\tau}
\end{array}\right) .
$$

The eigenvalues of this matrix for all $U$ in $\mathcal{U}$, are:

$$
\lambda_{1}(U)=-\left(\frac{\gamma(\gamma-1) e}{\tau^{2}}\right)^{1 / 2}, \quad \lambda_{2}(U)=0, \quad \lambda_{3}(U)=\left(\frac{\gamma(\gamma-1) e}{\tau^{2}}\right)^{1 / 2}
$$


We notice that to the second eigenvalue corresponds to a linearly degenerate characteristic field: for all $U$ in $\mathcal{U}, \nabla \lambda_{k}(U) \cdot r_{k}(U)=0$. This is the reason why we will look for viscous profiles approaching some 1-shocks and some 3-shocks. Indeed, a viscous profile cannot approach a contact discontinuity because a necessary condition for a viscous profile to exist is [4]:

$$
[\mathcal{F}(U)]<\sigma[\mathcal{E}(U)]
$$

where, $\mathcal{E}$ is a strictly convex entropy such that $B(U)$ is dissipative for it. $\mathcal{F}$ is an entropy flux associated to $\mathcal{E}$ and $\sigma$ is the speed of the discontinuity. Now, for a contact discontinuity $[\mathcal{F}(U)]=\sigma[\mathcal{E}(U)]$ occurs.

\subsection{Study of the Dynamical System}

The operator $B(U)$ is a bidimensional range operator and we can show that $\mathcal{U}=\operatorname{ker} B(U) \oplus \Im B(U)$. The eigenvalues of $B(U)$ (4) are: $\kappa, \mu, 0$, with respective eigenvectors, with $z>0$ :

$$
s_{1}(U)=(0,0,1)^{t}, \quad s_{2}(U)=(0,1, z)^{t}, \quad s_{3}(U)=(1,0,0)^{t} .
$$

Then $\operatorname{ker} B(U)=\mathbb{R} s_{3}(U)$. Projecting the algebraic equations onto $\operatorname{ker} B(U)$ gives an affine manifold of $\mathcal{U} \subset \mathbb{R}_{+}^{*} \times \mathbb{R} \times \mathbb{R}_{+}^{*}$ :

$$
V\left(u_{g} ; \sigma\right)=\left\{(\tau, z, e) \in \mathcal{U} \subset \mathbb{R}_{+}^{*} \times \mathbb{R} \times \mathbb{R}_{+}^{*}, \tau=\tau_{g}+\frac{z_{g}-z}{\sigma}\right\} .
$$

By doing this, the dynamical system projected on $V\left(u_{g} ; \sigma\right)$ writes:

$$
\left\{\begin{array}{l}
\mu z^{\prime}=\frac{\sigma(\gamma-1) E}{\sigma \tau_{g}+z_{g}-z}-\frac{\sigma(\gamma-1) z^{2}}{2\left(\sigma \tau_{g}+z_{g}-z\right)}-p_{g}-\sigma\left(z-z_{g}\right) \\
\kappa E^{\prime}+z z^{\prime}(\mu-\kappa)=\frac{\sigma(\gamma-1) E z}{\sigma \tau_{g}+z_{g}-z}-\frac{\sigma(\gamma-1) z^{3}}{2\left(\sigma \tau_{g}+z_{g}-z\right)}-p_{g} z_{g}-\sigma\left(E-E_{g}\right)
\end{array}\right.
$$

Note that: $\operatorname{ker} B(U) \oplus \Im B(U)=\operatorname{ker} B(U) \oplus V\left(u_{g} ; \sigma\right)$.

We set on $w=\left(w_{1}, w_{2}\right)^{t}=(z, E)^{t}$ and $\xi=(x-\sigma t) / \varepsilon$, too. Now, we shall suppose that the shock's speed $\sigma$ will be nonconstant. To avoid any confusion we shall call it $s$ to differentiate it to the constant value $\sigma$. Set:

$$
\tilde{B}(w(\xi), s)=\left(\begin{array}{cc}
\mu & 0 \\
(\mu-\kappa) z & \kappa
\end{array}\right),
$$

and

$$
\left\{\begin{array}{l}
\bar{F}=\left(\begin{array}{l}
f_{2} \\
f_{3}
\end{array}\right): \mathcal{U} \subset \mathbb{R}_{+}^{*} \times \mathbb{R} \times \mathbb{R}_{+}^{*} \rightarrow \mathbb{R}^{2}, \\
(\tau, z, E) \mapsto\left\{\begin{array}{l}
f_{2}(\tau, z, E) \\
f_{3}(\tau, z, E)
\end{array}\right.
\end{array}\right.
$$


And $F$ such that:

$$
F(z, E, s)=\left(\begin{array}{l}
f_{2}\left(\frac{z_{g}-z}{s}+\tau_{g}, z, E\right) \\
f_{3}\left(\frac{z_{g}-z}{s}+\tau_{g}, z, E\right)
\end{array}\right) \text {. }
$$

We shall note by $F_{s}$ the function, with a fixed $s$, defined as $F_{s}(z, E)=F(z, E, s)$ i.e., we have to resolve the following dynamical system (with the limit conditions imposed above):

$$
\tilde{B}(w, s) w^{\prime}=F(w, s)-\bar{F}\left(u_{g}\right)-s\left(w-w_{g}\right) .
$$

Note that for $w_{g}=w$ the RHS of the previous equation vanishes. Then, on the manifold $V\left(u_{g} ; s\right)$ :

$$
d_{\xi} w(\xi)=\tilde{B}(w(\xi), s)^{-1}\left(F(w(\xi), s)-\bar{F}\left(u_{g}\right)-s\left(w(\xi)-w_{g}\right)\right)=g(w(\xi), s) .
$$

Now, we increase the dynamical system, that gives on $V\left(u_{g} ; s\right)$,

$$
\left(\begin{array}{l}
w \\
s
\end{array}\right)^{\prime}=\left(\begin{array}{c}
g(w, s) \\
0
\end{array}\right)=G(w, s)
$$

Setting $P=\left(w_{g}, \lambda_{k}\left(u_{g}\right)\right)$ with $k \neq 2$ we want to linearize the new system around $P$ :

$$
d_{\xi}\left(\begin{array}{c}
w(\xi) \\
s(\xi)
\end{array}\right)=G(w(\xi), s(\xi))
$$

We deduce that:

$d G(P)=\left(\begin{array}{cc}\tilde{B}\left(w_{g}, \lambda_{k}\left(u_{g}\right)\right)^{-1}\left(\frac{\partial F}{\partial w}\left(w_{g}, \lambda_{k}\left(u_{g}\right)\right)-\lambda_{k}\left(u_{g}\right) I_{2}\right) & -\tilde{B}\left(w_{g}, \lambda_{k}\left(u_{g}\right)\right)^{-1} w_{g} \\ 0 & 0\end{array}\right) \in \mathcal{M}_{3}(\mathbb{R})$.

We make, now, some elementary calculus that will be useful in the sequel. To begin, we determine the eigenelements of $(\partial F / \partial w)\left(w_{g}, \lambda_{k}\left(u_{g}\right)\right)$ where:

$$
\frac{\partial F}{\partial w}\left(w_{g}, s\right)=\left(\begin{array}{cc}
\frac{(\gamma-1)}{s \mu \tau_{g}^{2}}\left(e_{g}-z_{g} \tau_{g} s\right) & \frac{(\gamma-1)}{\mu \tau_{g}} \\
\frac{(\gamma-1)}{s \tau_{g}^{2}} e_{g}\left(z_{g}+s \tau_{g}\right)+\frac{(1-\gamma) z_{g}^{2}}{\tau_{g}} & \frac{(\gamma-1) z_{g}}{\tau_{g}}
\end{array}\right) .
$$


We find that:

$$
\operatorname{Sp}\left(\frac{\partial F}{\partial w}\left(w_{g}, \lambda_{k}\left(u_{g}\right)\right)\right)=\left\{\bar{\lambda}_{1}\left(w_{g}, \lambda_{k}\left(u_{g}\right)\right)=\lambda_{k}\left(u_{g}\right), \bar{\lambda}_{2}\left(w_{g}, \lambda_{k}\left(u_{g}\right)\right)=\frac{1-\gamma}{\gamma} \lambda_{k}\left(u_{g}\right)\right\}
$$

Furthermore, for the first eigenvalue $\lambda_{1}(P)$ we determine a left and a right eigenvector:

$$
\left\{\begin{array}{l}
\bar{l}_{1}\left(w_{g}, \lambda_{k}\left(u_{g}\right)\right)=\left(-z_{g}+\left(\frac{\gamma}{\gamma-1} e_{g}\right)^{1 / 2}, 1\right), \\
\bar{r}_{1}\left(w_{g}, \lambda_{k}\left(u_{g}\right)\right)=\left(1, z_{g}+\left(\frac{\gamma}{\gamma-1} e_{g}\right)^{1 / 2}\left(\gamma^{1 / 2}-\gamma^{-1 / 2}\right)\right)^{t} .
\end{array}\right.
$$

In the same manner we obtain $(\partial F / \partial w)\left(w_{g}, \lambda_{k}\left(u_{g}\right)\right)-\lambda_{k}\left(u_{g}\right) I d_{2}$ :

$$
\frac{\partial F}{\partial w}\left(w_{g}, s\right)-s I d_{2}=\left(\begin{array}{cc}
\frac{(\gamma-1)}{s \mu \tau_{g}^{2}}\left(e_{g}-z_{g} \tau_{g} s\right)-s & \frac{(\gamma-1)}{\mu \tau_{g}} \\
\frac{(\gamma-1)}{s \tau_{g}^{2}} e_{g}\left(z_{g}+s \tau_{g}\right)+\frac{(1-\gamma) z_{g}^{2}}{\tau_{g}} & \frac{(\gamma-1) z_{g}}{\tau_{g}}-s
\end{array}\right),
$$

The eigenvalues are of course:

$$
\operatorname{Sp}\left(\frac{\partial F}{\partial w}\left(w_{g}, \lambda_{k}\left(u_{g}\right)\right)-\lambda_{k}\left(u_{g}\right) I d_{2}\right)=\left\{\bar{v}_{1}\left(w_{g}, \lambda_{k}\left(u_{g}\right)\right)=0, \bar{v}_{2}\left(w_{g}, \lambda_{k}\left(u_{g}\right)\right)=\frac{1-2 \gamma}{\gamma} \lambda_{k}\left(u_{g}\right)\right\} .
$$

The left and right eigenvectors associated to the zero eigenvalue are the same as those of $(\partial F / \partial w)\left(w_{g}, \lambda_{k}\left(u_{g}\right)\right)$.

Return to the study of $d G(10)$. Since:

$$
\begin{aligned}
\tilde{B}\left(w_{g}, s\right)^{-1} & \left(\frac{\partial F}{\partial w}\left(w_{g}, s\right)-s I d_{2}\right)= \\
& \frac{1}{\mu}\left(\begin{array}{cc}
\frac{(\gamma-1)}{s \mu \tau^{2}}\left(e_{g}-z_{g} \tau_{g} s\right)-s & \frac{(\gamma-1)}{\mu \tau} \\
\frac{s(\gamma-1) e}{s \tau^{2}}\left(e_{g} z_{g}-z_{g}^{2} \tau_{g} s+\operatorname{Pr} e_{g} s \tau_{g}\right)-z_{g} s(1-\operatorname{Pr}) & \frac{(\gamma-1)}{\tau_{g}}-s \operatorname{Pr}
\end{array}\right),
\end{aligned}
$$

then $d G\left(\left(w_{g}, s\right)\right)$ admits 0 as a double eigenvalue under the condition, with Pr fixed, that for $s=\lambda_{k}\left(u_{g}\right)$ :

$$
s^{2}(1+\operatorname{Pr}) \neq \frac{(\gamma-1) e_{g}}{\tau_{g}^{2}}
$$

and as simple eigenvalue $\left((\gamma-1) e_{g} / \tau_{g}^{2}\right)-s^{2}(1+\operatorname{Pr})$. Consequently, for $s=\lambda_{k}\left(u_{g}\right)$ the eigenvalues of $d G\left(\left(w_{g}, s\right)\right)$ are 0 twice and $(1-(1+\operatorname{Pr}) \gamma) p_{g}$ one time. This last eigenvalue is always strictly negative because of the positivity of the Prandtl number and of $\gamma-1$. Furthermore the eigenvectors associated to 0 are $(\overrightarrow{0}, 1)^{t}$ and $\left(\bar{r}_{1}\left(w_{g}, \lambda_{k}\left(u_{g}\right)\right), 0\right)^{t}$. 
Fundamental remark We search for an orbit joining $u_{d}$ to $u_{g}$ since $\left(u_{g}, u_{d} ; s\right)$ constitutes a $k$-shock. We can, following this, assert that $u_{d}$ belongs to $\Gamma_{g}^{+}\left(u_{g}\right)$ or $\Gamma_{g}^{-}\left(u_{g}\right)$ the connected components of the Hugoniot locus $\Gamma_{k}\left(u_{g}\right)$ associated to the $k^{\text {th }}$ characteristic.

Now, we can apply the center manifold theory presented in [4]. Consequently, since $d G\left(w_{g}, \lambda_{k}\left(u_{g}\right)\right)$ admits zero as eigenvalue of multiplicity 2, and a simple nonzero eigenvalue $\tilde{\lambda}_{2}\left(w_{g}, \lambda_{k}\left(u_{g}\right)\right)$, and that,

$$
\operatorname{ker} d G(P)=\operatorname{Vect}\left((\overrightarrow{0}, 1)^{t},\left(\bar{r}_{1}\left(w_{g}, \lambda_{k}\left(u_{g}\right)\right), 0\right)^{t}\right)
$$

where

$$
\bar{r}_{1}\left(w_{g}, \lambda_{k}\left(u_{g}\right)\right)=\left(1, z_{g}+\left(\frac{\gamma}{\gamma-1} e_{g}\right)^{1 / 2}\left(\gamma^{1 / 2}-\gamma^{-1 / 2}\right)\right)^{t},
$$

then the center manifold theorem states [3] that a bidimensional manifold invariant under the flow exists and is tangent in $w_{g}$ to $\operatorname{ker} d G(P)$, denoted by $W_{c}$. This manifold can be parametrized by:

$$
\left(\begin{array}{l}
w \\
s
\end{array}\right) \mapsto\left(\begin{array}{c}
s \\
x=\bar{l}_{1}(P) \cdot\left(w-w_{g}\right)
\end{array}\right),
$$

where $\bar{l}_{1}(P)$ is a left eigenvector of $d F_{\lambda_{k}\left(u_{g}\right)}\left(w_{g}\right)$. Futhermore, this manifold contains all the orbits remaining in a neighborhood of $w_{g}$. The critical points of $G$ are given by $\left(w_{g}, s\right)$ where $s \in \mathbb{R}$, and by $\left(w, \sigma\left(u_{g}, w\right)\right)$ :

$$
\left\{\left(w_{g}, s\right), s \in \mathbb{R}\right\} \cup\left\{\left(w, \sigma\left(u_{g}, w\right)\right) / w \in \tilde{\Gamma}_{k}\left(u_{g}\right)\right\} \subset W_{c},
$$

where $\tilde{\Gamma}_{k}\left(u_{g}\right)$ is the projection of the Hugoniot locus $\Gamma_{k}\left(u_{g}\right)$ on the manifold $V\left(u_{g} ; \sigma\right)$. As it has been remarked above, the Hugoniot locus leaving from $u_{g}$ allows a parametrization (cf [2], page 149). Then, using this result on the center manifold, we obtain the following parametrization (we recall that $\bar{\lambda}_{1}\left(w_{g}\right)=\lambda_{k}\left(u_{g}\right)$ ):

$$
\sigma\left(u_{g}, w\right)-\lambda_{k}\left(u_{g}\right) \sim \frac{1}{2} \bar{l}_{1}\left(w_{g}, \lambda_{k}\left(u_{g}\right)\right) \cdot\left(w-w_{g}\right)
$$

Therefore, the fixed points of the dynamical system are given by, $\left\{w_{g}\right\} \times \mathbb{R}$ and $\left(w, \sigma\left(u_{g}, w\right)\right)$ where $\sigma \sim \lambda_{k}\left(u_{g}\right)-x / 2$. These two curves are transverse. Now, if we freeze $s$, the flow of the dynamical system is itself transverse to these two curves and then cut into two fixed points. We can add that the flow is orthogonal to $\{x=0\}$. In order to see it, it is sufficient to observe, as it will be done earlier, that the flow is described, with $\alpha$ a positive constant, by the differential equation:

$$
\frac{d x}{d \xi}=\alpha \cdot x+o\left(x^{2}\right),
$$


Then, for every couple $\left(w_{g}, \sigma\left(u_{g}, w_{d}\right)\right),\left(w_{d}, \sigma\left(u_{g}, w_{d}\right)\right)$, with $\sigma\left(u_{g}, w_{d}\right)$ close to $\lambda_{k}\left(u_{g}\right)$ there exists a heteroclinic orbit of the dynamical system joining these two states. Finally, we must determine the direction of the motion along the orbit. To this end, it is necessary to evaluate the sign of the derivative with respect to $x$ in $x=0$ of the following differential equation:

$$
\frac{d x}{d \xi}=h(\sigma, x)
$$

where:

$$
w-w_{g}=x \cdot \bar{r}_{1}\left(w_{g}, \lambda_{k}\left(u_{g}\right)\right)+o\left(x^{2}\right),
$$

since $W_{c}$ contains $\left\{w_{g}\right\} \times \mathbb{R}$ and is tangent to $\left(\bar{r}_{1}\left(w_{g}, \lambda_{k}\left(u_{g}\right)\right), 0\right) \in \operatorname{ker} d G(P)$. Now, $h$ is given by $h(s, x)=\bar{l}_{1}\left(w_{g}, \lambda_{k}\left(u_{g}\right)\right) \cdot \tilde{B}\left(w_{g}, \lambda_{k}\left(u_{g}\right)\right)^{-1} \cdot\left(F(w, s)-\bar{F}\left(u_{g}\right)-s\left(w-w_{g}\right)\right)$.

As for a fixed $s$ :

$$
F(w, s)-\bar{F}\left(u_{g}\right)-s\left(w-w_{g}\right)=\left(d F_{s}\left(u_{g}\right)-s I_{2}\right)\left(w-w_{g}\right)+o\left(\left|w-w_{g}\right|\right),
$$

then in (12),

$$
h(s, x)=\bar{l}_{1}\left(w_{g}, \lambda_{k}\left(u_{g}\right)\right) \cdot \tilde{B}\left(w_{g}, s\right)^{-1}\left(d F_{s}\left(w_{g}, s\right)-s I_{2}\right) \cdot\left(w-w_{g}\right)+o\left(\left|w-w_{g}\right|\right),
$$

where:

$$
\tilde{B}\left(w_{g}, \lambda_{k}\left(u_{g}\right)\right)^{-1}=\frac{1}{\mu}\left(\begin{array}{cc}
1 & 0 \\
z_{g}(1-\operatorname{Pr}) & \operatorname{Pr}
\end{array}\right) .
$$

Since $w-w_{g}=x \bar{r}_{1}\left(w_{g}, \lambda_{k}\left(u_{g}\right)\right)+o\left(x^{2}\right)$ then:

$$
\frac{d x}{d \xi}=\bar{l}_{1}\left(w_{g}, \lambda_{k}\left(u_{g}\right)\right) \cdot \tilde{B}\left(w_{g}, \lambda_{k}\left(u_{g}\right)\right)^{-1} \cdot \bar{r}_{1}\left(w_{g}, \lambda_{k}\left(u_{g}\right)\right) \cdot\left(\lambda_{k}\left(u_{g}\right)-s\right) x+o\left(x^{2}\right) .
$$

The dissipativity of $\tilde{B}$ allows us to deduce (precisions can be found in [4], or in [1]) that for all $U \in \mathcal{U}$ :

$$
\bar{l}_{1}(U) \cdot \tilde{B}(U)^{-1} \cdot \bar{r}_{1}(U)>0
$$

and notably

$$
\bar{l}_{1}\left(w_{g}, \lambda_{k}\left(u_{g}\right)\right) \cdot \tilde{B}\left(w_{g}, \lambda_{k}\left(u_{g}\right)\right)^{-1} \cdot \bar{r}_{1}\left(w_{g}, \lambda_{k}\left(u_{g}\right)\right)>0 .
$$

In fact we can check it directly:

$$
\begin{aligned}
\bar{l}_{1}\left(w_{g}, \lambda_{k}\left(u_{g}\right)\right) \cdot \tilde{B}\left(w_{g}, \lambda_{k}\left(u_{g}\right)\right)^{-1} \cdot \bar{r}_{1}\left(w_{g}, \lambda_{k}\left(u_{g}\right)\right)= & \frac{1}{\mu}\left(\left(\frac{\gamma}{\gamma-1} e_{g}\right)^{1 / 2}(1+\operatorname{Pr})\right. \\
& \left.+\operatorname{Pr}\left(1+\left(\frac{e_{g}}{(\gamma-1) \gamma}\right)^{1 / 2}\right)\right),
\end{aligned}
$$


or

$$
\begin{aligned}
\bar{l}_{1}\left(w_{g}, \lambda_{k}\left(u_{g}\right)\right) \cdot \tilde{B}\left(w_{g}, \lambda_{k}\left(u_{g}\right)\right)^{-1} \cdot \bar{r}_{1}\left(w_{g}, \lambda_{k}\left(u_{g}\right)\right)= \\
\frac{1}{\mu}\left(\frac{\gamma}{\gamma-1} e_{g}\right)^{1 / 2}\left(1+\operatorname{Pr}\left(\gamma^{1 / 2}-\gamma^{-1 / 2}\right)\right)>0 .
\end{aligned}
$$

This yields:

$$
\operatorname{sgn}\left(\partial_{x} h(s, 0)\right)=\operatorname{sgn}\left(\lambda_{k}\left(u_{g}\right)-s\right)
$$

Then, $w_{g}$ is a point of repulsion if, and only if, $\operatorname{sgn}\left(\lambda_{k}\left(u_{g}\right)-s\right)>0$. This condition is, in fact, is the Lax condition. As $w_{d}$ belongs to the projection in the Hugoniot locus, a regular path joining $w_{d}$ to $w_{g}$ exists. Since $\tau=\tau_{g}+\left(z_{g}-z\right) / \sigma$, we can extend the existence result to a path joining $u_{g}$ and $u_{d}$ : it is a viscous profile.

\subsection{Existence result}

Taking account the previous study we can now state the following result:

THEOREM 1 Consider the compressible Navier-Stokes equations:

$$
u_{t}+f(u)_{x}=\varepsilon\left(B(u) u_{x}\right)_{x}
$$

where:

$$
B(u)=\left(\begin{array}{ccc}
0 & 0 & 0 \\
0 & \mu & 0 \\
0 & (\mu-\kappa) z & \kappa
\end{array}\right) .
$$

(1) Let $u_{d}$ belonging to the Hugoniot locus $\Gamma_{k}\left(u_{g}\right)$ of $u_{g}$ with $k \neq 2$. If $s$ is the speed of the discontinuity such that (for $k=3$ ):

- $\left(\tau_{g}, e_{g}\right) \in\left\{(\tau, e) \in \tilde{\mathcal{U}} \subset\left(\mathbb{R}_{+}^{*}\right)^{2}, e>s^{2} \tau^{2} / \gamma(\gamma-1)\right\}$.

- $\left(\tau_{d}, e_{d}\right) \in\left\{(\tau, e) \in \tilde{\mathcal{U}} \subset\left(\mathbb{R}_{+}^{*}\right)^{2}, e<s^{2} \tau^{2} / \gamma(\gamma-1)\right\}$.

- $u_{g}$ and $u_{d}$ close enough.

A viscous profile joining $u_{g}$ and $u_{d}$ exists.

(2) Mutually, if $u_{d}$ belongs to the Hugoniot locus in a neighborhood of $u_{g}$ and if a viscous profile exists which joins these two states then $\left(u_{g}, u_{d} ; s\right)$ represents a nonstationary Lax k-shock.

\section{References}

[1] E. Lorin (2001). Sur la stabilité de modèles en mécanique des fluides numérique dans le contexte volumes finis. Thèse de l'Ecole Normale Supérieure de Cachan, France.

[2] C. Dafermos (2000). Hyperbolic Conservation Laws in Continuum Physics. Springer, C.S.M.

[3] C. Dafermos (1996). Differential Equations and Dynamical Systems. Springer, T.A.M. 
[4] D. Serre (1996). Systemes de lois de conservation. I: Hyperbolicite, entropies, ondes de choc in Fondations. Diderot Editeur, Paris.

[5] D. Serre (1999). Stabilit L1 des chocs et de deux types de profils, pour des lois de conservation scalaires. Séminaire EDP de l'X.

[6] H. Freistühler, C. Fries and C. Rohde (2001). Existence, bifurcation, and stability of profiles for classical and non-classical shock waves. Ergodic theory, analysis, and efficient simulation of dynamical systems, 287-309, 814, Springer, Berlin, 2001.

[7] D. Gilbarg (1951). The existence and limit behavior of the one-dimensional shock layer. Amer. J. Maths, 73. 